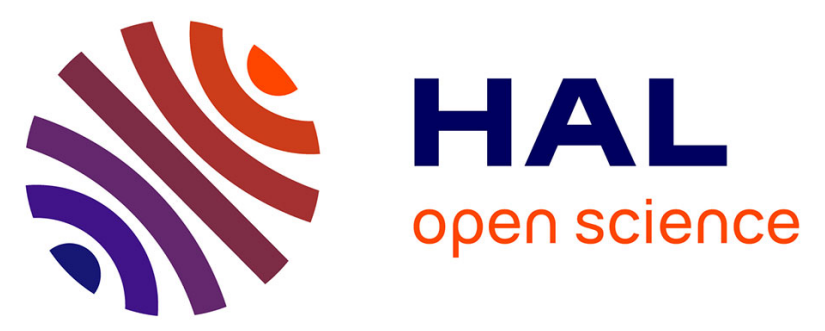

\title{
Model calculation of the magnetic induction generated by magnetic nanoparticles flowing into a microfluidic system. Performance analysis of the detection
}

Kamel Fodil, Matthieu Denoual, Christophe Dolabdjian, A. Treizebre, Vincent Senez

\section{To cite this version:}

Kamel Fodil, Matthieu Denoual, Christophe Dolabdjian, A. Treizebre, Vincent Senez. Model calculation of the magnetic induction generated by magnetic nanoparticles flowing into a microfluidic system. Performance analysis of the detection. IEEE Transactions on Magnetics, 2014, 50, pp.1-8. 10.1109/TMAG.2013.2291546 . hal-00980048

\section{HAL Id: hal-00980048 https://hal.science/hal-00980048}

Submitted on 18 Apr 2014

HAL is a multi-disciplinary open access archive for the deposit and dissemination of scientific research documents, whether they are published or not. The documents may come from teaching and research institutions in France or abroad, or from public or private research centers.
L'archive ouverte pluridisciplinaire HAL, est destinée au dépôt et à la diffusion de documents scientifiques de niveau recherche, publiés ou non, émanant des établissements d'enseignement et de recherche français ou étrangers, des laboratoires publics ou privés. 


\title{
Model Calculation of the Magnetic Induction Generated by Magnetic Nanoparticles Flowing Into a Microfluidic System: Performance Analysis of the Detection
}

\author{
Kamel Fodil ${ }^{1,2,4}$, Matthieu Denoual ${ }^{1,3,4}$, Christophe Dolabdjian ${ }^{1,2,4}$, \\ Anthony Treizebre ${ }^{5}$, and Vincent Senez ${ }^{5}$ \\ ${ }^{1}$ Normandie University, Caen F-14032, France \\ ${ }^{2}$ Université de Caen Basse-Normandie, Caen 14000, France \\ ${ }^{3}$ École Nationale Supérieure d'Ingénieurs de Caen, Caen F-14032, France \\ ${ }^{4}$ Centre National de la Recherche Scientifique, Caen F-14032, France \\ ${ }^{5}$ Université de Lille Nord de France, IEMN, Lille 59653, France
}

This paper presents a mathematical model of the detection of the magnetic nanoparticles (MNPs) flowing into a microchannel. To validate the model, the experimental measurements were performed using a ferrofluid and a giant magneto impedance microwire made up of CoFeSiBNb alloy as a sensor. The good agreement between theory and measurement implies the possibility of using the numerical simulation to analyze the system performance for the MNPs detection. In addition, a model calculation was performed, which evaluates the optimal signal-to-noise ratio of the detection system as a function of its main parameters and according to the magnetic induction induced by the MNPs.

Index Terms_Giant magneto impedance (GMI) microwire, magnetic nanoparticles (MNPs), microchannel.

\section{INTRODUCTION}

$\mathbf{M}$ AGNETIC nanoparticles (MNPs) are particles with diameters up to $50 \mathrm{~nm}$ made of iron, cobalt, or nickel [1]. They are of great interest for a wide range of biotechnological and biomedical applications involving the detection, the transport, and the immobilization of MNPs or magnetically tagged biological entities. Among the specific applications that should be mentioned, there are: the magnetic particle imaging [2], the magnetic resonance imaging contrast enhancement, drug/gene delivery, cell separation, biosensing, or hyperthermia cancer treatment [3]. For most of the applications, a high-sensitivity magnetic sensor is required for the detection of the MNPs. Several types of magnetic sensors have been used for the detection such as giant magnetoresistive (GMR) sensors [4], spin-valve sensors [5], fluxgate [6], SQUID [7], Hall sensors [8], pickup coil [9], and giant magneto impedance (GMI) [10].

The integration into a so-called microfluidic microsystem for the detection of flowing nanoparticles often leads to small sensors such as GMR [11], spin-valve [12], or GMI [13]-[16]. The technical challenge of the detection of flowing MNPs is to develop detection systems that are sensitive enough to detect a single MNP or clusters of nanoparticles, ideally in a continuous flow. Sensor performance is not the only condition to improve the MNP detection limit. Indeed, the entire system configuration surrounding the sensor is important. The aim of this paper is to describe a model, which accurately predicts the impact of some parameters of the system on the MNP

Manuscript received July 16, 2013; revised October 8, 2013; accepted November 9, 2013. Date of publication November 19, 2013; date of current version April 4, 2014. Corresponding author: K. Fodil (e-mail: kamel.fodil@ensicaen.fr).

Color versions of one or more of the figures in this paper are available online at $\mathrm{http}: / /$ ieeexplore.iee.org.

Digital Object Identifier 10.1109/TMAG.2013.2291546 detection performance (some parameters are not investigated as flow rate and the size of MNP). First, a general setup for the detection of MNPs in microchannel is explained in Section II. For a better understanding of the analytical model, the magnetic induction produced by the MNPs is described in Section III. In Section IV, the numerical calculation considering the experimental conditions is presented. The validation of the model with numerical and experimental comparison is carried out in Section V. Finally, a reduced model for the evaluation of the signal-to-noise ratio (SNR) of the system as a function of main parameters is presented in Section VI.

\section{General Setup for the Detection of the MNPS INTO A MICROCHANNEL}

The detection of MNPs requires the application of an external magnetic field to induce their magnetization and a magnetic sensor to sense this stray magnetic induction.

Usually, the external magnetic excitation induction $\vec{B}_{\text {ext }}$ is perpendicular to the sensitive axis of the sensor [6], [10], [17] to prevent its saturation and enable high dynamic range measurements. This perpendicular configuration is preferred also for the detection of nanoparticles flowing into a microchannel (see Fig. 1). The MNPs can be found under various forms, as powders or suspension in a liquid medium. For the purposes of detection in microfluidic system, plugs of liquid of MNPs or ferrofluid (fluid containing a dispersion of MNPs in suspended form or as colloids) are injected into a microchannel.

\section{Calculation of the Magnetic Induction Produced by THE MNPS IN THE MicrochanNel}

In this section, a theoretical model of the stray magnetic induction generated by the presence of MNPs is evaluated according to the configuration shown in Fig. 1. 


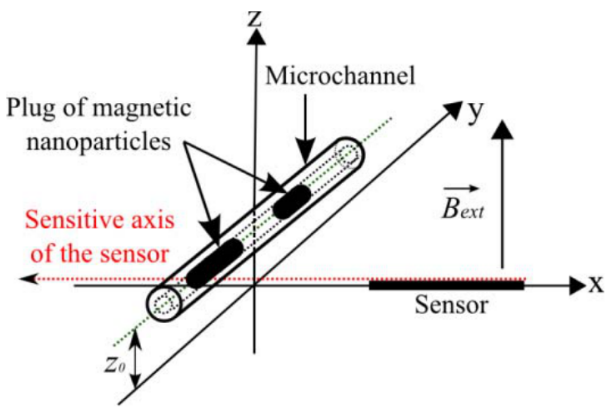

Fig. 1. Sketch view of the detection setup with plugs of MNPs flowing into a microchannel.

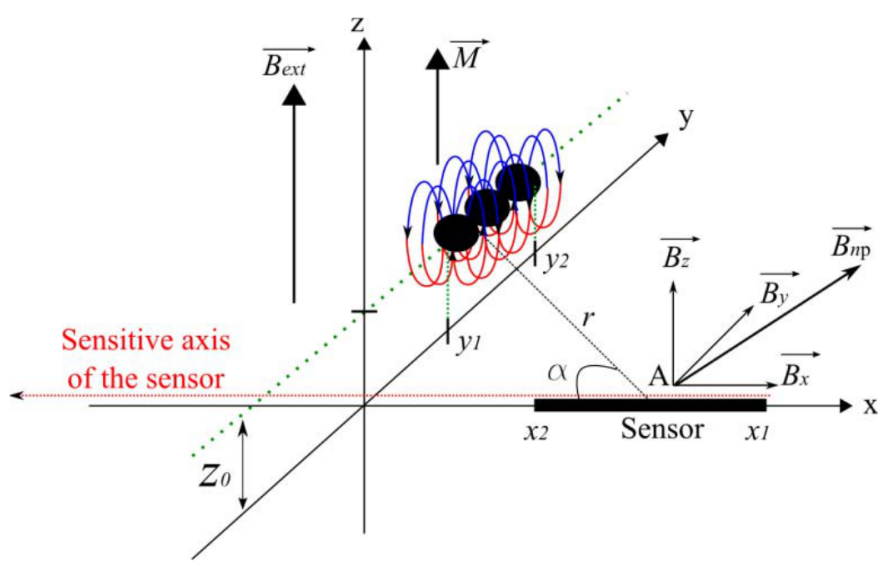

Fig. 2. Magnetic induction created at point $\mathrm{A}$, in space, by the magnetic moment $\vec{M}$ of the MNPs. The three spheres represent, as an example, MNPs of a plug flowing into a microchannel.

In the model, Cartesian coordinates are chosen as they are more relevant. The MNPs are magnetized by $\vec{B}_{\text {ext }}$ along the $z$-axis (see Figs. 1 or 2 ). The expression of the magnetic induction $\vec{B}_{\mathrm{np}}$ created at a point $\mathrm{A}$, in space, by a magnetic moment $\vec{M}$ of the MNPs is given by

$$
\overrightarrow{B_{\mathrm{np}}}=\frac{\mu_{0}}{4 \pi} \overrightarrow{\mathrm{grad}}\left(\frac{\vec{M} \cdot \vec{r}}{r^{3}}\right) \text {. }
$$

If we consider a single MNP, $\vec{r}$ becomes the distance of that MNP to point A of the sensor. The magnetic moment of the MNPs depending on the external magnetic field $\vec{H}_{\text {ext }}$ is given by

$$
\vec{M}=\chi_{m} \cdot C \cdot V_{t} \cdot \overrightarrow{H_{\mathrm{ext}}}
$$

where $\chi_{m}, C$ and $V_{t}$ are the molar magnetic susceptibility, the concentration of the MNPs, and the total volume occupied by the MNPs, respectively.

The magnetic sensor senses the small magnetic induction variation along the $x$-axis, $\overrightarrow{B_{x}}$, produced by the MNPs placed into an external magnetic excitation induction. From (1), the magnetic induction $B_{x}$ is written as

$B_{x}=\frac{\mu_{0}}{4 \pi} \cdot \frac{\partial}{\partial x} \frac{M \cdot z}{\left(x^{2}+y^{2}+z^{2}\right)^{\frac{3}{2}}}=\frac{\mu_{0} M}{4 \pi} \cdot \frac{-3 z x}{\left(x^{2}+y^{2}+z^{2}\right)^{\frac{5}{2}}}$.

We define an elementary magnetic moment, $d m=M_{L} d y$. $M_{L}$ is defined as a linear magnetic moment, according to
$M=\int_{y 1}^{y 2} M_{L} d y$. Hence

$$
M_{L}=\frac{M}{\left(y_{2}-y_{1}\right)} .
$$

The average magnetic induction throughout a wire-shaped sensor with a length of $\left(x_{2}-x_{1}\right)$ is obtained by a mathematical integration along the $x$-axis, such as

$$
<B_{d m}>=\frac{\mu_{0}}{4 \pi} \cdot \frac{3 Z_{0}}{x_{2}-x_{1}} \int_{x 1}^{x 2} \frac{-x}{\left(x^{2}+y^{2}+Z_{0}^{2}\right)^{\frac{5}{2}}} d x d m
$$

where $Z_{0}$ is the altitude difference between the two surfaces of magnetic sensor and the microchannel. The total magnetic induction, $B_{T}$, sensed by the magnetic sensor is valuated from the integration of (5) along the displacement $y$-axis of the MNPs. It yields

$$
B_{T}=M_{L} \frac{\mu_{0}}{4 \pi} \cdot \frac{3 Z_{0}}{x_{2}-x_{1}} \int_{y 1}^{y 2} \int_{x 1}^{x 2} \frac{-x}{\left(x^{2}+y^{2}+Z_{0}^{2}\right)^{\frac{5}{2}}} d x d y .
$$

Finally, (6) gives the expression of the total magnetic induction sensed by the magnetic sensor and due to the displacement of the MNP plugs as

$$
\begin{aligned}
B_{T}= & \frac{\chi_{m} \cdot C \cdot V_{t} \cdot Z_{0} \cdot B_{\mathrm{ext}}}{4 \pi\left(x_{2}-x_{1}\right)\left(y_{2}-y_{1}\right)} \\
& \left(\begin{array}{l}
\frac{1}{x_{2}^{2}+Z_{0}^{2}} \cdot\left(\frac{y_{2}}{{\sqrt{x_{2}^{2}+y_{2}^{2}+Z_{0}}}^{2}}-\frac{y_{1}}{{\sqrt{x_{2}^{2}+y_{1}^{2}+Z_{0}}}^{2}}\right) \\
-\frac{1}{x_{1}^{2}+Z_{0}^{2}} \cdot\left(\frac{y_{2}}{{\sqrt{x_{1}^{2}+y_{2}^{2}+Z_{0}}}^{2}}-\frac{y_{1}}{{\sqrt{x_{1}^{2}+y_{1}^{2}+Z_{0}}}^{2}}\right)
\end{array}\right)
\end{aligned}
$$

where $x_{1}$ and $x_{2}$ are the location of the wire-shaped magnetic sensor and $y_{1}$ and $y_{2}$ are the location of the MNP plugs into the microchannel.

\section{Numerical Calculation Considering THE EXPERIMENTAL CONDITIONS}

In this section, the given model, (7), is used to derive the behavior of the detection setup. The effects of the external magnetic induction and the volume, concentration, and susceptibility of the magnetic particles are straightforward, since the total magnetic induction changes linearly with them. Therefore, the behavior study focuses especially on the geometrical parameters. The sharp decay of the magnetic induction produced by the nanoparticles with the distance $\left(B_{x} \sim 1 / x^{4}\right)$ makes the distance between the microchannel and the magnetic sensor along the $x$-axis $\left(x_{2}\right)$ and the length $\left(x_{1}-x_{2}\right)$ key parameters. Both of them should be as small as possible to increase the value of the mean magnetic induction sensed by the GMI. Notice that only technological issues will limit the positioning of $x_{2}$ and $x_{1}$. Nevertheless, the study of the effect of the sensor length $\left(x_{1}-x_{2}\right)$ is not investigated as it induces changes in the transfer and noise of the sensor. Moreover, for the same length, these parameters (transfer and noise) change from one GMI microwire to another.

The relevant $y$-dimension is the length of the plug and the relevant $z$-dimension is $Z_{0}$. The impacts of those two parameters are illustrated, hereafter, through the numerical 


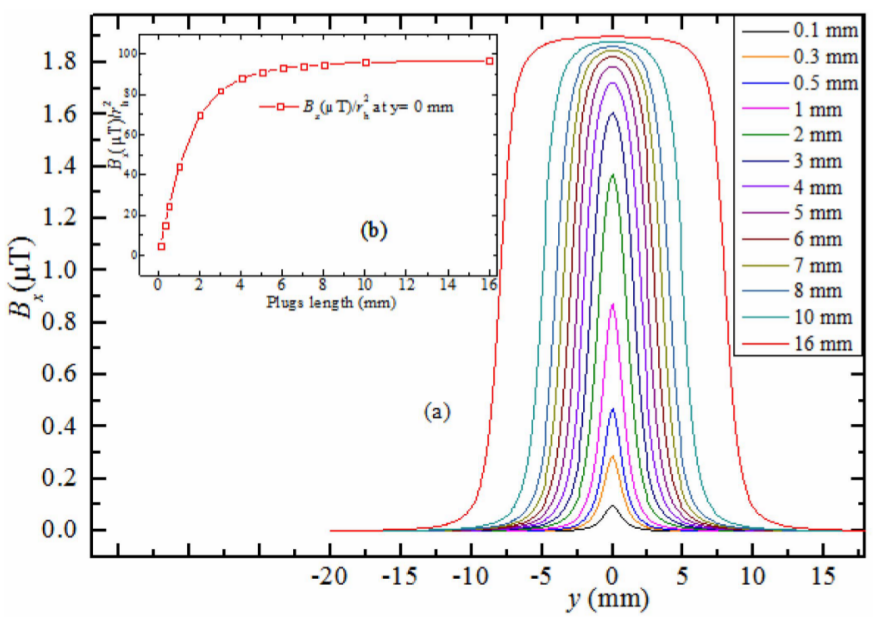

Fig. 3. (a) Theoretical magnetic induction, $B_{x}$, induced by the displacement of plugs of MNPs along the $y$-axis, given for different lengths $(0.1-16 \mathrm{~mm}$ long), $Z_{0}=400 \mu \mathrm{m}, x_{2}=800 \mu \mathrm{m}$, and $x_{1}=1.08 \mathrm{~cm}$. (b) Inset shows the maximum magnetic induction value as a function of the lengths of the plugs at the position $y=0$. Curves are normalized to the square of the microchannel radius.

application studies. For those numerical applications, we use the experimental parameters given in Section $\mathrm{V}$ to enable easier comparison $\left(C=10.6 \mathrm{~mol} / \mathrm{L}, \chi_{m}=0.066 \mathrm{emu} / \mathrm{mol}\right.$, $V_{t}=18 \mathrm{~nL}, B_{\mathrm{ext}}=6.8 \mathrm{mT}$, and the radius of the microchannel $\left.r_{h}=140 \mu \mathrm{m}\right)$.

\section{A. Study According to Length of the Plug $\left(y_{2}-y_{1}\right)$}

Fig. 3(a) shows the magnetic induction $B_{x}$ induced for different lengths of plug $(0.1-16 \mathrm{~mm})$ during a displacement along the $y$-axis. Fig. 3(b) shows the maximum magnetic induction obtained when the plugs are centered along the sensitive axis of the sensor $(y=0 \mathrm{~mm})$. We notice that the curves are normalized to the square of the microchannel radius, $r_{h}$. The curve behaviors show that the relation between the total magnetic induction and the length of the plug becomes nonlinear as the length of the plugs increases. Considering the experimental setup parameters and until the length of the plugs of the MNPs increases $>3 \mathrm{~mm}$, the signal exhibits a small increase. This behavior is consistent with experimental results given in Section V.

\section{B. Study According to the Sensor Liftoff $\left(Z_{0}\right)$}

The sensor liftoff, $Z_{0}$, between the microchannel and the sensitive axis of the sensor is of crucial importance. Either when $Z_{0}$ is zero or tends to be infinite, the sensed signal tends toward zero. Between those two limits, an optimum positioning exists. This optimum can be easily determined, when punctual magnetic moment and sensor are considered. With the given configuration, the optimum cannot be analytically determined easily. This consideration drives the study according to the parameter $Z_{0}$.

Fig. 4 shows the behavior of the total magnetic induction according to $Z_{0}$ value for several distances between the microchannel and the magnetic sensor $\left(x_{2}\right)$. For each distance case, an optimum $Z_{0}$ clearly appears. The precise positioning

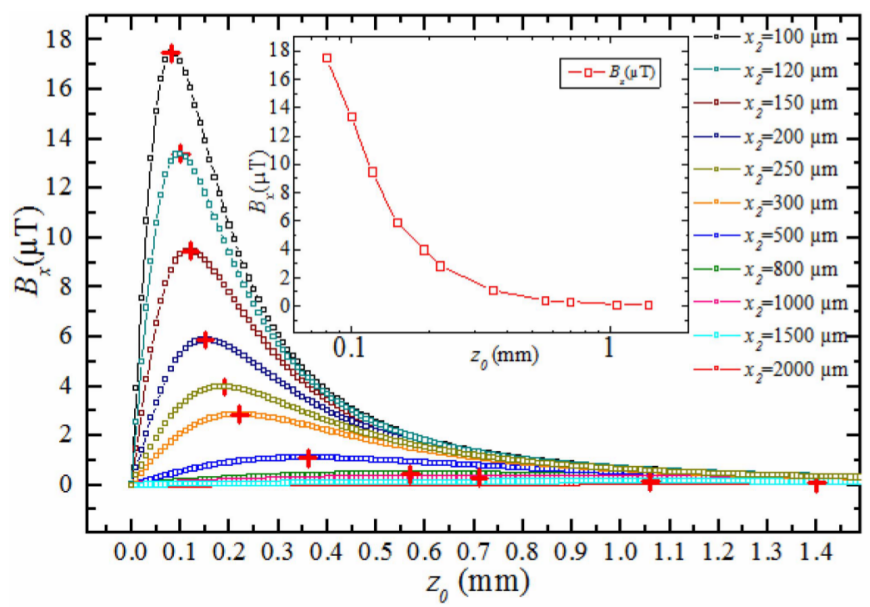

Fig. 4. Magnetic induction, $B_{x}$, evaluated for a plug length $\left(y_{2}-y_{1}\right)=$ $0.3 \mathrm{~mm}$ (corresponding to a volume of $18 \mathrm{~nL}$ ), depending on the sensor liftoff, $Z_{0}$, and for different distances between the magnetic sensor and the microchannel, $x_{2}$. Red crosses: the optimum for each case. They are replotted in the inset.

of the sensor becomes more important as the distance between the sensor and the microchannel decreases because of the slopes of the magnetic induction versus $Z_{0}$ becoming steeper. Anyway, bringing the sensor closer to the microchannel always improves the sensed signal.

Fig. 4 clearly shows that there is an optimum $Z_{0}$ for a given $x_{2}$. In our measurement setup (see Section V), the $x_{2}$ and $Z_{0}$ are $\sim 800$ and $400 \mu \mathrm{m}$, respectively. With such conditions, the magnetic induction is $0.3 \mu \mathrm{T}$ (see Fig. 5). For the same volume, an optimal setting of $x_{2}$ and $Z_{0}$ yields a signal 60 times greater than previously studied (two orders of magnitude) for values of $x_{2}$ of $100 \mu \mathrm{m}$ and $Z_{0}$ of $80 \mu \mathrm{m}$, as shown in Fig. 4.

In practice, technological issues limit the optimized realization of the setup. A GMI microwire is used as a sensor for the experimental part. In practice, it is difficult to have a real mastery over the distance $x_{2}$. First, because the micropositionning of the wire close to the microchannel is technically challenging. Second, the soldering for the electrical contact renders a part of the GMI microwire inoperative that cannot be easily quantified.

\section{VALidation OF THE Model}

This section compares the experimental results with (7). Refer to [18] for a detailed description of the experimental setup.

The measurement involves injecting plugs of MNPs into the microchannel. A video camera and an acquisition card record the displacement of the plug of nanoparticles and the measured signal, respectively. The simultaneous recording enables the study the correlation between the measured magnetic signal and the position of the MNP plugs into the microchannel.

Fig. 5 shows a typical signal acquired during the displacement of plugs, in this case, with two successive passages of the MNPs liquid plugs of the volumes of $18 \mathrm{~nL}\left(y_{2}-y_{1}=\right.$ $0.3 \mathrm{~mm})$ and $55 \mathrm{~nL}\left(y_{2}-y_{1}=0.9 \mathrm{~mm}\right)$. Plugs are spaced by 


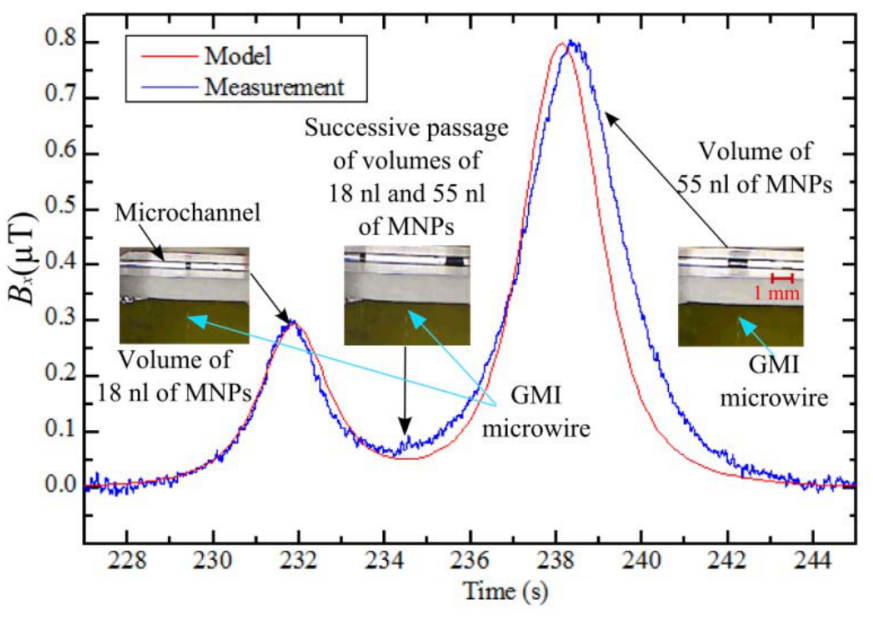

Fig. 5. Numerical and experimental comparison of the magnetic induction induced by the motion of two successive plugs of 0.3 and $0.9 \mathrm{~mm}$ long of ferrofluid and spaced by $4 \mathrm{~mm}$. The small pictures are extracted from the video recording.

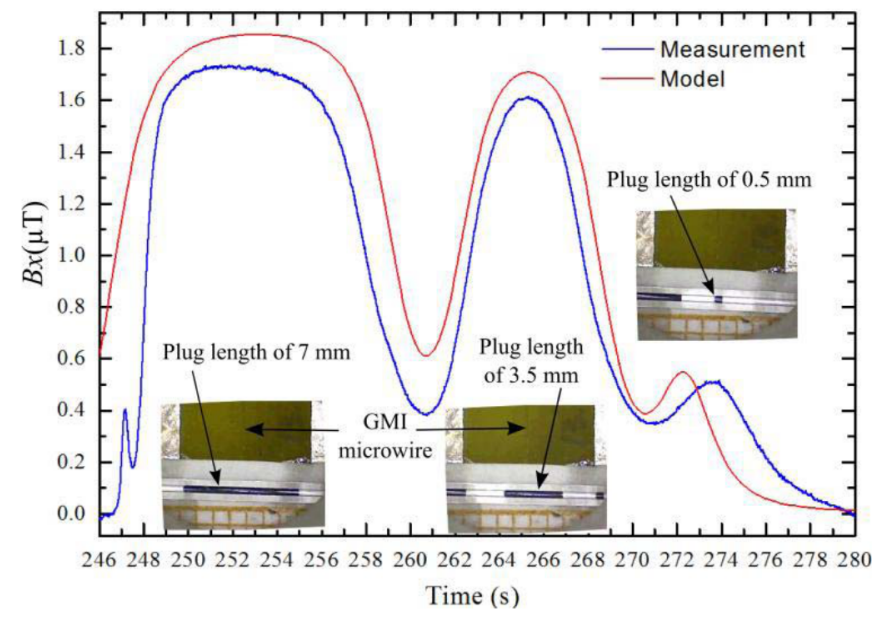

Fig. 6. Comparison between numerical and experimental $B_{x}$ signal induced by the passage of three successive ferrofluid plugs of length $7,3.5$, and $0.5 \mathrm{~mm}$, respectively.

a distance of $4 \mathrm{~mm}$ and have an average speed of $0.4 \mathrm{~mm} / \mathrm{s}$. The signal increases as the plugs approach and decreases when they move away. The amplitude of the signal is higher when the volume/length of the plug is bigger.

Fig. 5 also plots the curve obtained with (7) and gives the parameters of the experiment. It shows the good agreement between the model and the experimental results. The slight mismatch between the model and the measure in the case of the $55 \mathrm{~nL}$ plug $(0.9 \mathrm{~mm}$ plug $)$ is explainable by the difference of speed between both plugs. Indeed, the speed of the $18 \mathrm{~nL}$ plug $(0.3 \mathrm{~mm}$ plug) was considered for the time-to-space conversion yielding an error for the other plug. Fig. 6 shows the case of big plugs, as studied previously and shown in Fig. 3.

The measurement of Fig. 6 shows that effectively there exists a weak variation of the magnetic induction between the plugs of 3.5 and $7 \mathrm{~mm}$ ( 215 and $430 \mathrm{~nL}$, respectively) despite of a volume twice bigger. These results strengthen even more

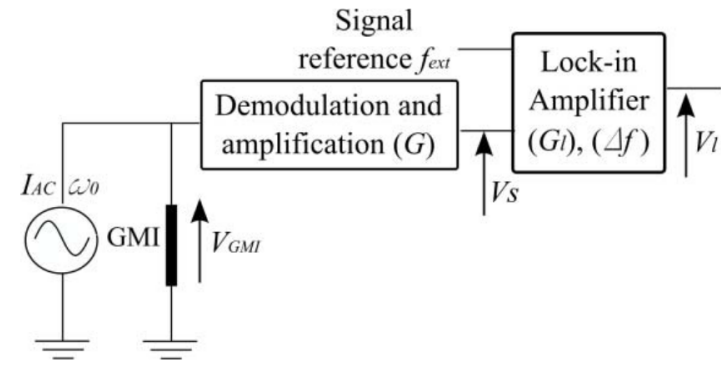

Fig. 7. Basic sketch view of the electronic measurement chain.

the adequacy of the theoretical model. We notice that the slight mismatch is due to the different plug speeds. In Fig. 6, the plugs have an average speed of $0.6 \mathrm{~mm} / \mathrm{s}$ and are spaced by a distance of $2 \mathrm{~mm}$.

The good agreement between the numerical calculation and the experimental results validates the analytical model, and implies the possibility of using this model to analyze the system performance for the MNPs detection.

As a rule, for the major part of the applications, the challenge is to detect concentrations and volumes of MNPs as small as possible. Therefore, after considering the optimization of the magnetic induction response of the MNPs (Fig. 4), considering the electronics and noise optimization to improve the SNR is equally important.

The detection threshold may be reduced by the noise of the sensor, the noise of the electronics, or by a general optimization of the detection system.

\section{SNR OF THE SENSING SyStem}

The SNR of the system depends on several parameters such as the noise of the sensor, the electronics, the intensity of the magnetic signal induced by the MNPs, and so on. This section deals with the study and the configuration of the system to obtain an optimal SNR for the MNPs detection.

\section{A. Expression of the $S N R$}

At first, a study of the whole electronics measurement chain with the GMI microwire ( $40 \mu \mathrm{m}$ diameter and $1 \mathrm{~cm}$ long wire) (see Fig. 7) is performed to calculate the output signal of the chain and deduce the SNR of the system as a function of its main parameters. A detailed description of the system can be found in [18].

The GMI microwire sensing is based on impedance variations due to magnetic induction. The impedance variations are converted into voltage through an ac biasing at a proper dc magnetic bias working point [19] followed by an amplitude demodulation. Then, the lock-in detector enables us to track the sensor output signal related to the external magnetic induction excitation. The usual magnetoimpedance signal variation produced by the magnetic induction generated by the MNPs is given by

$$
Z\left(\omega_{0}, B_{\text {bias }}+b_{\mathrm{np}}\right)=\frac{Z\left(\omega_{0}, B_{\text {bias }}\right)}{\mu_{0}}+\left.\frac{\partial Z\left(\omega_{0}, B\right)}{\partial B}\right|_{B} \cdot b_{\mathrm{np}} .
$$


The voltage appearing across the GMI microwire is then

$$
V_{\mathrm{GMI}}(t)=Z\left(\omega_{0}, B_{\mathrm{bias}}+b_{\mathrm{np}}\right) \cdot I_{\mathrm{AC}} \cdot \sin \left(\omega_{0} t\right)
$$

where $\omega_{0}$ and $I_{\mathrm{AC}}$ are the angular frequency and the amplitude of the excitation current, respectively. $B_{\text {bias }}$ is the dc bias magnetic induction, which sets the field working point for the GMI microwire. $b_{\mathrm{np}}$ is a small magnetic induction variation induced by the MNPs such as $b_{\mathrm{np}}=B_{T} \cdot \sin \left(2 \pi f_{\mathrm{ext}} t\right)$. We notice that $B_{T}$ is equivalent as (7) and $f_{\text {ext }}$ is the frequency of the external induction field, $B_{\text {ext }}$.

After demodulation and amplification, the output voltage $V_{S}(t)$ is given by

$$
V_{s}(t)=\frac{Z\left(\omega_{0}, B_{\mathrm{bias}}\right)}{\mu_{0}} \cdot G \cdot I_{\mathrm{AC}}+G \cdot T_{r} \cdot b_{\mathrm{np}}
$$

where $G$ is the gain of the amplifier and $T_{r}(\mathrm{~V} / \mathrm{T})$ is the sensitivity of the GMI microwire expressed by $T_{r}=\left.\frac{\partial Z\left(\omega_{0}, B\right)}{\partial B}\right|_{B} \cdot I_{\mathrm{AC}}$. An equivalent electronic voltage noise, $e_{n}(t)$, appears, too, at the output voltage of (10), such as

$$
\begin{aligned}
V_{s}(t)= & \frac{Z\left(\omega_{0}, B_{\text {bias }}\right)}{\mu_{0}} \cdot G \cdot I_{\mathrm{AC}} \\
& +G \cdot T_{r} \cdot B_{T} \cdot \sin \left(2 \pi f_{\mathrm{ext}} t\right)+G \cdot e_{n}(t)
\end{aligned}
$$

with

$$
e_{n}(t)=p(t) \cdot \sin \left(2 \pi f_{\mathrm{ext}} t\right)-q(t) \cdot \cos \left(2 \pi f_{\mathrm{ext}}\right)
$$

and $\overline{p^{2}(t)}=\overline{q^{2}(t)}=e_{n}^{2}$, where $p(t)$ and $q(t)$ are the in- and quadrature-phase temporal noise components, respectively. Finally, from (12), the total output signal (using a synchronous demodulation with a centered square reference signal of a frequency, $f_{\text {ext }}$ ) is given by

$$
V_{l}=\frac{2}{\pi} \cdot\left(G \cdot G_{l} \cdot T_{r} \cdot B_{T}+G \cdot G_{l} \cdot e_{n}\right)
$$

where $e_{n}=\sqrt{\overline{p^{2}(t)}} . G_{l}$ is the gain of the lock-in amplifier.

Thus, from (13), the SNR of the measurement chain is expressed as $\mathrm{SNR}=\operatorname{Tr} \cdot B_{T} / e_{n}$. From there, it ultimately yields

$$
\mathrm{SNR}=\frac{B_{T}}{b_{n}} .
$$

The input equivalent noise considers the white noise and flicker noise contributions as expressed by

$$
b_{n}=\sqrt{2} \cdot \sqrt{\left(\int_{f_{\mathrm{ext}}-\frac{\Delta f}{2}}^{f_{\mathrm{ext}}+\frac{\Delta f}{2}}\left(\frac{a^{2}}{\left(f_{\mathrm{ext}}^{b}\right)^{2}}+c^{2}\right) \cdot d f\right)}
$$

where $a, b$, and $c$ are the constants associated to the noise model and $\Delta f$ is the bandwidth of the lock-in amplifier. The bandwidth $\Delta f$ is selected as a function of the speed rate of the MNPs flowing into the microchannel.

The excitation Helmholtz coil of the MNPs plays a significant role in the optimization of the SNR of the system. It is characterized by geometrical size and number of turns that defines its transfer and its electrical parameters inductance, $L_{c}$, and resistance, $R_{c}$. The transfer in (T/A) of the Helmholtz coils is expressed by

$$
T_{r_{\text {coil }}}=\left(\frac{4}{5}\right)^{3 / 2} \cdot \mu_{0} \cdot \frac{N}{r_{c}}
$$

where $N, r_{C}$ is the number of turns and $r_{C}$ is the radius of the coil. The electrical parameters are given by empirical equations [20] as

$$
L_{c}=\frac{0.08 \cdot\left(2 \cdot r_{c}\right)^{2} \cdot N^{2}}{6 \cdot r_{c}+9 \cdot l_{c}+10 \cdot t_{c}} \cdot 10^{-6}
$$

and

$$
R_{c}=\frac{\rho \cdot 2 \pi \cdot r_{c} \cdot N}{s_{w}}
$$

where $l_{c}$ and $t_{c}$ are the length and the thickness of the Helmholtz coil, respectively. $\rho$ is the resistivity of the copper and $s_{w}$ is the section of the copper wire such as

$$
s_{w}=\frac{\pi \cdot d_{w}^{2}}{4}
$$

where $d_{w}$ is the diameter of the wire used for the coil design.

The electrical parameters set the bandwidth of the excitation coils, $B_{d}$, as well as the resistor in series with the coils $R_{s}$ following:

$$
B_{d}=\frac{2 \cdot R_{c}+R_{s}}{2 \pi \cdot 2 \cdot L_{c}} .
$$

From (14) and (16) a general equation, which calculates the optimum SNR of the system as a function of main parameters, including the model of the magnetic induction created by the displacement of the MNPs into the microchannel, is given as

$$
\begin{aligned}
\mathrm{SNR} & =\left(\frac{\chi_{m} \cdot C \cdot V_{t} \cdot\left(\frac{4}{5}\right)^{\frac{3}{2}} \cdot \mu_{0} \cdot \frac{N \cdot I}{r_{c}}}{4 \pi \cdot b_{n}}\right) \\
& \cdot f\left(x_{1}, x_{2}, y_{1}, y_{2}, Z_{0}\right)
\end{aligned}
$$

where $I$ is the current flowing in the Helmholtz coil.

\section{B. Improvement of the SNR}

On the one hand, the noises of the sensor and the acquisition chain have to be minimized. Appropriate biasing and conditioning of the GMI sensor allow reaching physical limits of the noise of such sensors. On the other hand, the signal can be increased.

Indeed, the higher the magnetic excitation induction is, the greater the response signal of the MNPs is, until the field corresponding to the saturation of the magnetization of the MNPs. In the case of the sample used in the experiments, this saturation field is $50 \mathrm{mT}$.

Thus, a high transfer, through a larger number of turns $(N)$ (16), of the excitation Helmholtz coil should be a criterion of choice. However, as a counterpart, the bandwidth (20) will decrease because of increased inductance with $N$. Such characteristics can be a limitation of the system depending on the displacement speed of the plugs. The bandwidth of the excitation coil also affects the noise. Indeed, for sensors such as the GMI microwire, the low-frequency noise is significant. 
Therefore, if the magnetic induction excitation, $B_{\text {ext }}$, is at low frequency, the SNR decreases at constant magnetic excitation induction. Ideally, to compensate for this effect, the magnetic excitation induction is increased. It means increasing the current flowing in the Helmholtz coil, I. However, we notice that the current cannot be increased indefinitely in real life.

In addition, the frequency, $f_{\text {ext }}$, is selected considering the noise limitation of the GMI microwire and the bandwidth of the excitation coils. As $L_{c}$ and $R_{c}$ are fixed by the design of the coils (17), (18), the SNR is optimized by adjusting the resistor in series $R_{S}$ and thus the excitation frequency is chosen accordingly. It yields $f_{\mathrm{ext}}=B_{d}$ (while wanting to be as far as possible from the $1 / f$ noise corner of the sensor). The resistor $R_{S}$ in series with the excitation coils affects the bandwidth but also the current passing through the coils and therefore the transfer of the Helmholtz coil. However, the impacts are opposite. Increasing the bandwidth through the increase of $R_{S}$ reduces the transfer and affects the SNR, because of signal reduction. The bandwidth required for the detection application as well as power issues lower limit the value of the excitation frequency. The power involved $P$ is also calculated as a function of the variation of $R_{S}$ and therefore $f_{\text {ext }}$. The power is expressed by

$$
P=\left(R_{S}+R_{c}\right) \cdot I^{2} \text {. }
$$

The first power issue is related to the power supplied by the system and that has to be dissipated (technological limitation). It is introduced in the model through a maximum current value from the current supply, $I_{\text {supply }}^{\max }$.

The second power issue comes from the maximum current density achievable with the wires of the Helmholtz coil (physical limitation). It depends on the material used, here copper, and is expressed by a maximum current, $I_{\text {phys }}^{\max }$, according to the diameter of the copper wire.

The minimal value of those two currents limits the realistic operation of the system!

We set, in the numerical valuation, the maximum supply current of the excitation source, $I_{\text {supply }}^{\max }(=3$ A) and the maximum current density, $I_{\text {phys }}^{\max }\left(=5.10^{6} \cdot s_{w}\right)$.

Thus, there exists an optimum between the maximum intensity of the magnetic excitation induction, the excitation frequency, and the noise of the sensor.

\section{Behavior of the SNR Versus System Parameters}

This section illustrates some behavioral trends of SNR of the system depending on its size represented by the radius of the Helmholtz coil and its power consumption.

The model is based on the following:

1) all the parameters of the magnetic induction created by the MNPs (the concentration $C$, the volume $V_{\text {np }}$ of the MNPs, the sizes and positions of the sensor $x_{1}$ and $x_{2}$, the length of the plug $y_{2}-y_{1}$ and the liftoff, $Z_{0}$ );

2) the electronic parameters (the excitation voltage, the resistor in series with the exciting coils, the bandwidth of the synchronous detection, and so on);

3 ) the geometry of the excitation Helmholtz coil (the radius, the length, the thickness, the number of turns of the coils, and the diameter of the wire used);

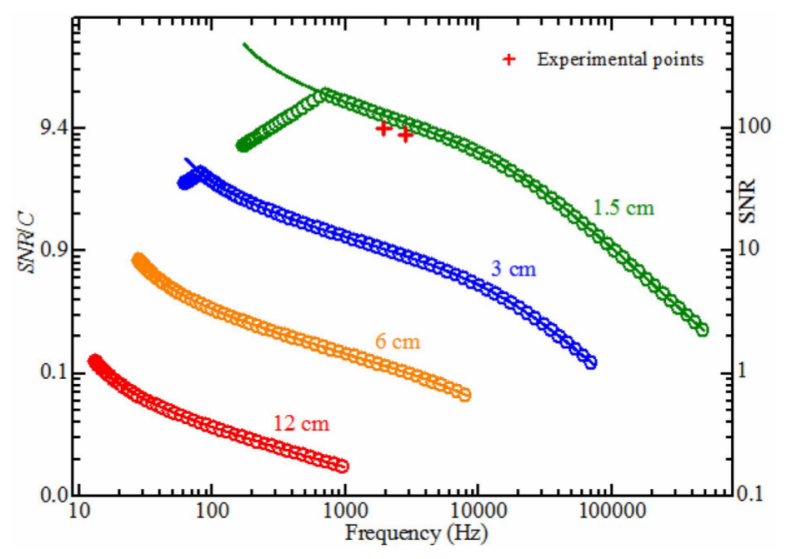

Fig. 8. SNR of the system as a function of frequency of $\vec{B}_{\text {ext }}$ for a given volume of the MNPs of $18 \mathrm{~nL}\left(y_{2}-y_{1}=0.3 \mathrm{~mm}\right)$, for different Helmholtz coil radii $(1.5,3,6$, and $12 \mathrm{~cm})$, for a same transfer (17) and for a same excitation voltage of $10 \mathrm{~V}$, with (small circles) and without (solid line) limitation of the current. Curves are normalized to the concentration, $C$.

4) the noise model of the sensor used.

Then, the model calculates the following:

1) the magnetic induction according to (7);

2) the SNR of the system and the power as a function of the excitation frequency or $R_{S}$ according to (21).

In the following, some illustrative and representative cases are exemplified, for a better understanding of the issue. Figs. 8-10 show the comparison of the SNR and the implemented powers for different sizes or radii of the excitation coils with the same transfer, for different excitation voltages, and with the noise model of the GMI microwire used for the experiments.

On all the following figures, both possibilities with (small circles) or without (solid line) current limitation are taken considered.

Fig. 8 shows that the SNR decreases according to the radius of the excitation coils for the same excitation voltage source of $10 \mathrm{~V}$. The increase of the volumes of the excitations coils leads to the decrease of their bandwidth and their transfer.

To increase their transfer, the number of turns should be increased and, in this case, the bandwidth decreases and the resistor of the coils increases, which causes the decrease of the current injected in the excitation coils. To prevent this fall of SNR with the increased volume of the excitation coils, the excitation voltage should be increased according to the radius of the excitation coils. In this way, bigger coils can reach the same order of magnitude of SNR as the small coils $(1.5 \mathrm{~cm})$, as shown in Fig. 9. The latter shows the SNR as a function of the frequency for different volumes of the excitation coils with a suited voltage excitation (to obtain a same SNR), for different radius of excitation coils. In all cases, an optimum SNR of $\sim 200$ is achieved (for the optimum frequency of $700 \mathrm{~Hz}$ ). Nevertheless, to obtain this order of magnitude, the required powers that are implemented are huge, as shown in Fig. 10.

Fig. 10 shows an evaluation of the power required for various radii of the excitation coils and the resulting SNR. Considering the experimental setup, with coils of $1.5 \mathrm{~cm}$ and 


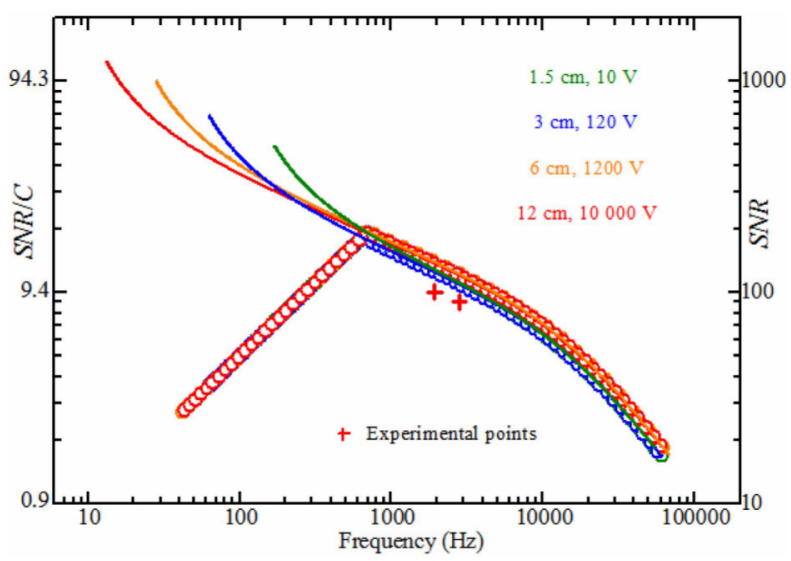

Fig. 9. SNR of the system as a function of frequency of $\vec{B}_{\text {ext }}$ for a given volume of the MNPs of $18 \mathrm{~nL}\left(y_{2}-y_{1}=0.3 \mathrm{~mm}\right)$, for different excitation coils' radius $(1.5,3,6$, and $12 \mathrm{~cm})$, for the same transfer and for different voltage excitations (suited to each radius coil, 10, 120, 1200, and $10000 \mathrm{~V}$, respectively), with (small circles) and without (solid line) limitation of the current. Curves are normalized to the concentration, $C$.

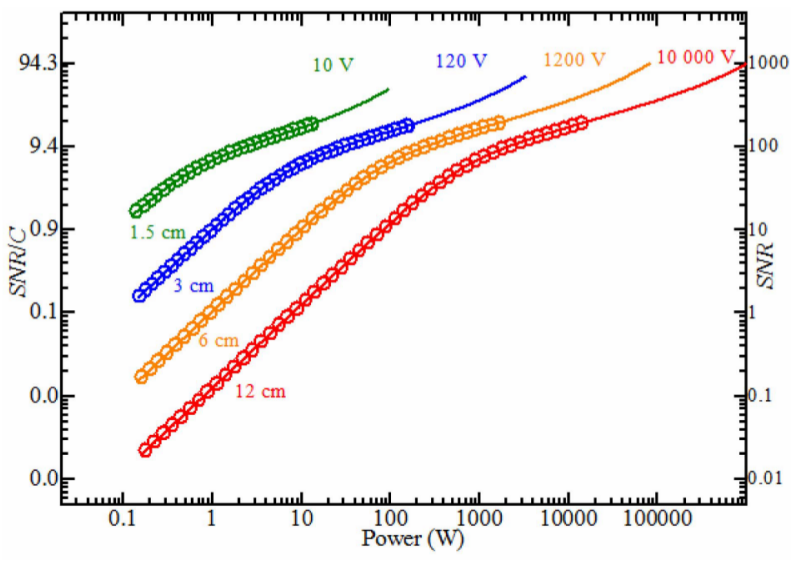

Fig. 10. Concentration normalized SNR of the system as a function of the power for a given volume of the MNPs of $18 \mathrm{~nL}\left(y_{2}-y_{1}=0.3 \mathrm{~mm}\right)$, for different excitation coils' radius $(1.5,3,6$, and $12 \mathrm{~cm})$, for the same transfer and for different voltages excitations (adjusted for each radius coil, 10, 120, 1200 , and $10000 \mathrm{~V}$, respectively), with (small circles) and without (solid line) limitation of the current.

with a power of only $2 \mathrm{~W}$, the SNR is $\sim 100$. Whereas, the SNR would be less than one with a coil radius of $12 \mathrm{~cm}$ and $2 \mathrm{~W}$ of power.

Fig. 11 shows powers involved as a function of the coils radius for defined SNR of $1,10,100$, and 200. To achieve an SNR of 100 with a coil radius of $12 \mathrm{~cm}$, a power of $\sim 2000 \mathrm{~W}$ is necessary. Meanwhile, this power value is disproportionate for this microfluidic detection application. It should be noted that the design of the excitation coils has to be carried out according to the application and/or the volume of the sample to be analyzed.

If the detection of MNPs injected into veins of mice is the goal, there is no other choice than to increase the volume of excitation coils (otherwise, it will be impossible to put the mice between the coils). These interesting issues related to the power supplied in applications dealing with the detection of MNPs are well known.

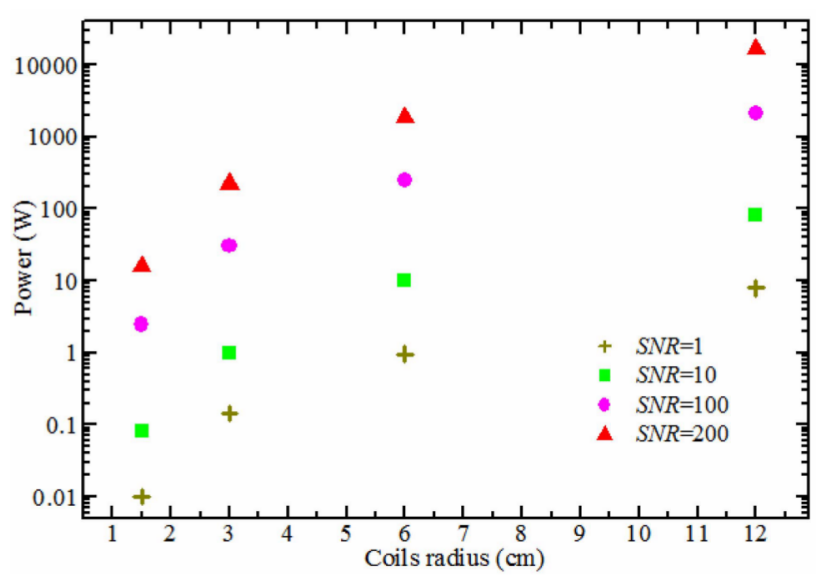

Fig. 11. Power of the system as a function of the radius of the excitation coils $(1.5,3,6$, and $12 \mathrm{~cm})$ with the same transfer, for a volume of MNPs of $18 \mathrm{~nL}$ and for different constant SNRs of 1, 10, 100, and 200.

\section{CONCLUSION}

With a setup of magnetic particles detection, an analytical model of magnetic induction produced by the MNPs in the microchannel was developed.

The experimental results strengthen the adequacy of the theoretical model and imply the possibility of using the numerical calculation to analyze system performance, using system approach, for the detection of MNP plugs. Henceforward, this model can be used to predict the detection of a particular concentration or volume of MNPs depending on the configuration of the system and consider the possible improvements to be carried out.

To illustrate the usefulness of the model, let us consider the use of MNPs as a label for blood vessels imaging or angiography application for a human hand.

In that case, coils with radius of $1.5 \mathrm{~cm}$ are too tiny compared with a human hand, even if the goal is to detect a small volume of MNPs related to the diameter and the length of the vein (assimilated to the microchannel in this paper). Coils with radius of $6 \mathrm{~cm}$ or higher are more appropriate. Therefore, the power involved is higher. In addition, for this application, the MNPs are injected in vivo, therefore a maximum safety dosage has to be respected; rated at $40 \mu \mathrm{mol}(\mathrm{Fe}) / \mathrm{L}$ in [21]. Thereafter, with all this information, analyzing system performance can be performed with the proposed model and feasible enhancements considered. Assuming that a liftoff $Z_{0}$ in this case (a human hand) is $\sim 0.5 \mathrm{~cm}$ and $x_{2}$ tends toward zero. A rough optimization can be done for the estimation of the feasibility of the detection in vivo.

Let us use a $6 \mathrm{~cm}$ radius coil with a transfer of $50 \mathrm{mT} / \mathrm{A}$ (instead of $12 \mathrm{mT} / \mathrm{A}$ in the paper's setup) and a wire diameter $d_{w}$ of $2 \mathrm{~mm}$ (instead of $0.6 \mathrm{~mm}$ in the paper's setup). In the presented setup, the noise floor of the GMI microwire is $100 \mathrm{pT} / \sqrt{\mathrm{Hz}}$. Knowing the state of the art of the GMI sensor development, for example, [22], an improvement factor of 100 can be achieved for the noise figure. With these rough enhancements, using a voltage excitation of $2000 \mathrm{~V}$, a plug of MNPs, with a concentration of $40 \mu \mathrm{mol}(\mathrm{Fe}) / \mathrm{L}$, and a volume 
of $180 \mathrm{~nL}$ ( $3 \mathrm{~mm}$ long), can be sensed with an SNR of two and a power of $\sim 5000 \mathrm{~W}$. This result might still be optimized by considering other parameter enhancements. In real life, it is more complicated than it sounds. Actually, the MNPs will flow freely in the vein and they would not be restricted in a plug. This will result in a particle spread and the concentration will decrease. Meanwhile, the model developed in this paper helps to investigate and optimize the performance of the system.

\section{ACKNOWLEDGMENT}

This work was supported in part by MXT Montréal for providing the GMI microwires and in part by the CNANO Nanotrans Program.

\section{REFERENCES}

[1] S. P. Gubin, Y. A. Koksharov, G. B. Khomutov, and G. Y. Yurkov, "Magnetic nanoparticles: Preparation, structure and properties," Russian Chem. Rev, vol. 74, no. 6, pp. 489-520, Jun. 2005.

[2] E. U. Saritas, P. W. Goodwill, L. R. Croft, J. J. Konkle, K. Lu, B. Zheng, et al., "Magnetic particle imaging (MPI) for NMR and MRI researchers.," J. Magn. Reson., vol. 229, pp. 116-126, Dec. 2012.

[3] A. M. G. C. Dias, A. Hussain, A. S. Marcos, and A. C. A. Roque, "A biotechnological perspective on the application of iron oxide magnetic colloids modified with polysaccharides," Biotechnol. Adv., vol. 29 , no. 1, pp. 142-155, 2011.

[4] C. Marquina, J. M. de Teresa, D. Serrate, J. Marzo, F. A. Cardoso, D. Saurel, et al., "GMR sensors and magnetic nanoparticles for immunochromatographic assays," J. Magn. Magn. Mater., vol. 324, no. 21, pp. 3495-3498, Oct. 2012.

[5] G. Li, V. Joshi, R. L. White, S. X. Wang, J. T. Kemp, T. Jennifer, et al., "Detection of single micron-sized magnetic bead and magnetic nanoparticles using spin valve sensors for biological applications," J. Appl. Phys., vol. 93, no. 10, pp. 10-13, 2012.

[6] J. Dieckhoff, M. Schilling, and F. Ludwig, "Fluxgate based detection of magnetic nanoparticle dynamics in a rotating magnetic field," Appl. Phys. Lett., vol. 99, no. 11, pp. 112501-1-112501-3, 2011.

[7] M. S. Katsura, T. Yasuda, K. Hirano, A. Mizuno, and S. Tanaka, "Development of a new detection method for DNA molecules," Sci. Technol., vol. 14, no. 12, pp. 1131-1134, 2001.

[8] L. Ejsing, M. F. Hansen, and A. Menon, "Magnetic microbead detection using the planar Hall effect," J. Magn. Magn. Mater., vol. 293, no. 1, pp. 677-684, 2005.
[9] B. Gleich and J. Weizenecker, "Tomographic imaging using the nonlinear response of magnetic particles," Nature, vol. 435, no. 7046, pp. 1214-1217, Jun. 2005.

[10] H. Chiriac, M. Tibu, A.-E. Moga, and D. D. Herea, "Magnetic GMI sensor for detection of biomolecules," J. Magn. Magn. Mater, vol. 293, pp. 671-676, May 2005.

[11] N. Pekas, M. D. Porter, M. Tondra, A. Popple, and A. Jander, "Giant magnetoresistance monitoring of magnetic picodroplets in an integrated microfluidic system," Appl. Phys. Lett., vol. 85, no. 20, pp. 4783-4785, 2004.

[12] H. A. Ferreira, N. Feliciano, D. L. Graham, and P. P. Freita, "Effect of spin-valve sensor magnetostatic fields on nanobead detection for biochip applications," J. Appl. Phys., vol. 97, no. 10, pp. 10Q904-1-10Q904-6, 2005.

[13] A. Garcia-Arribas, F. Martinez, E. Fernandez, I. Ozaeta, G. V. Kurlyandskaya, A. V. Svalov, et al., "GMI detection of magnetic-particles concentration in continuous flow," Sens. Actuators A, Phys., vol. 172, no. 1, pp. 103-108, Mar. 2011

[14] M. Denoual, M. Harnois, S. Saez, C. Dolabdjian, and V. Senez, "Microfluidic microsystem for magnetic sensing of nanoparticles with giant magneto-impedance technology," in Proc. 16th Int. Solid-State Sensors, Actuat. Microsyst. Conf., Jun. 2011, pp. 80-83.

[15] G. V. Kurlyandskaya, M. L. Sanchez, B. Hernando, V. M. Prida, P. Gorria, and M. Tejedor, "Giant-magnetoimpedance-based sensitive element as a model for biosensors," Appl. Phys. Lett., vol. 82, no. 18, pp. 3053-1-3053-5, 2003.

[16] H. Yang, L. Chen, C. Lei, J. Zhang, D. Li, Z. Zhou, et al., "Giant magnetoimpedance-based microchannel system for quick and parallel genotyping of human papilloma virus type 16/18," Appl. Phys. Lett., vol. 97, no. 4, pp. 043702-1-043702-3, 2012.

[17] P. P. Freitas and H. A. Ferreira, "Spintronic biochips for biomolecular recognition," Handbook Magn. Adv. Magn. Mater, vol. 4, pp. 1-29, Jan. 2007.

[18] K. Fodil, M. Denoual, C. Dolabdjian, M. Harnois, and V. Senez, "Dynamic sensing of magnetic nanoparticles in microchannel using GMI technology," IEEE Trans. Magn., vol. 49, no. 1, pp. 93-96, Jan. 2013.

[19] D. Ménard and A. Yelon, "Theory of longitudinal magnetoimpedance in wires," J. Appl. Phys., vol. 88, no. 1, pp. 379-393, 2000.

[20] M. Thompson, Inductance Calculation Techniques-Part II: Approximations and Handbook Methods. Columbus, OH, USA: Intelligent Motion, Dec. 1999.

[21] V. Dousset, C. Gomez, K. G. Petry, C. Delalande, and J. M. Caille, "Dose and scanning delay using USPIO for central nervous system macrophage imaging," Elsevier Sci., Magn. Reson. Mater. Phys., Biol. Med., vol. 8, no. 3, pp. 185-189, 1999.

[22] B. Dufay, S. Saez, C. Dolabdjian, A. Yelon, and D. Ménard, "Impact of electronic conditioning on the noise performance of a two-port network giant magnetoimpedance magnetometer," IEEE Sensors J., vol. 11, no. 6 , pp. 1317-1324, Jun. 2010. 